\title{
A STRONG LAW FOR LINEAR FUNCTIONS OF ORDER STATISTICS
}

\author{
BY W. R. VAN ZWET \\ University of Leiden
}

\begin{abstract}
A strong law of large numbers for linear combinations of order statistics is proved under integrability conditions only. Together with some straightforward extensions, the theorem generalizes previous results of Wellner, Helmers and Sen.
\end{abstract}

1. Introduction. Let $U_{1}, U_{2}, \cdots$ be random variables defined on a single probability space $(\Omega, Q, P)$ and suppose that $U_{1}, U_{2}, \cdots$ are independent and identically distributed (i.i.d.) according to the uniform distribution on $(0,1)$. For $N=1,2, \cdots, U_{1: N}<U_{2: N}<\cdots<U_{N: N}$ denote the ordered $U_{1}, \cdots, U_{N}$. Introduce Lebesgue measurable functions $J_{N}:(0,1) \rightarrow \mathbb{R}, N=1,2, \cdots$, a Borel measurable function $g:(0,1) \rightarrow \mathbb{R}$ and define $g_{N}:(0,1) \rightarrow \mathbb{R}, N=1,2, \cdots$, by

$$
g_{N}(t)=g\left(U_{[N t]+1: N}\right),
$$

where $[x]$ denotes the integer part of $x$. We adopt the convention that when integration is with respect to Lebesgue measure $\lambda$ on $(0,1)$, we shall write $\int f$ for $\int f d \lambda$. The range of integration will be $(0,1)$ unless explicitly indicated otherwise. For $1 \leqslant p \leqslant \infty, L_{p}$ is the Lebesgue space of measurable functions $f:(0,1) \rightarrow \mathbb{R}$ with finite norm $\|f\|_{p}=\left\{\int|f|^{p}\right\}^{1 / p}$ for $1 \leqslant p<\infty$ and $\|f\|_{\infty}=\operatorname{ess} \sup |f|$ for $p=\infty$.

The purpose of this note is to show that under integrability assumptions on $J_{N}$ and $g$,

$$
M_{N}=\int J_{N}\left(g_{N}-g\right)=\sum_{i=1}^{N} g\left(U_{i: N}\right) \int_{(i-1) / N}^{i / N} J_{N}-\int J_{N} g
$$

converges to zero for $N \rightarrow \infty$ with probability 1 (w.p. 1). If, moreover, $J_{N}$ converges in an appropriate sense to a function $J$ which shares the integrability properties of $J_{N}$, we prove that

$$
\tilde{M}_{N}=\int J_{N} g_{N}-\int J g
$$

also converges to zero w.p. 1 .

If $J_{N}(t)=c_{N, i}$ for $(i-1) / N<t \leqslant i / N, i=1, \cdots, N$, and $g=h \circ F^{-1}$ for a probability distribution function (df) $F$ on $\mathbb{R}$ and a Borel measurable function $h: \mathbb{R} \rightarrow \mathbb{R}$, then the joint distribution of $\int J_{N} g_{N}, N=1,2, \cdots$, is that of $N^{-1} \sum c_{N, i} h\left(X_{i: N}\right), N=1,2, \cdots$, where the $X_{i: N}$ are order statistics of a sequence of i.i.d. random variables with common df $F$. We are therefore concerned with the almost sure convergence of suitably standardized linear combinations of a function of order statistics.

Received September 25, 1978; revised May 22, 1979.

AMS 1970 subject classifications. Primary 60F15; secondary 62G30.

Key words and phrases. Strong law, order statistics. 
Previous results in this direction may be found in Wellner (1977), Helmers (1977) and Sen (1978). Wellner restricts attention to the case where $J_{N}(t)=c_{N, i}$ for $(i-1) / N<t \leqslant i / N, i=1, \cdots, N$, and assumes that $g$ is left continuous and of bounded variation on closed subintervals of $(0,1)$. He proves that $M_{N} \rightarrow 0$ w.p. 1 if numbers $b_{1}, b_{2}$ and $C$, as well as $\delta>0$ exist such that, for all $N$ and $t \in(0,1)$,

$$
\begin{aligned}
|g(t)| & \leqslant C t^{-1+b_{1}+\delta}(1-t)^{-1+b_{2}+\delta}, \\
\left|J_{N}(t)\right| & \leqslant C t^{-b_{1}}(1-t)^{-b_{2}}, \\
\int t^{1-b_{1}-\frac{1}{2} \delta}(1-t)^{1-b_{2}-\frac{1}{2} \delta} d|g| & <\infty,
\end{aligned}
$$

where $d|g|$ denotes integration with respect to the total variation measure induced by $g$. He shows that $\tilde{M}_{N} \rightarrow 0$ w.p. 1 under the additional assumption that $J_{N}$ converges to $J$ pointwise.

It is clear that Wellner's result will cover most cases that one is likely to come across in practice, the main flaw being that it just fails to contain the strong law for the sample mean, i.e., the case where $J_{N} \equiv 1$ and $g \in L_{1}$. This gap is closed in Helmers (1977) where it is shown that $\tilde{M}_{N} \rightarrow 0$ w.p. 1 for $c_{N, i}=J(i /(N+1)), J$ piecewise continuous and bounded and $g=F^{-1} \in L_{1}$.

For $b_{1}, b_{2} \in[0,1)$, Wellner's conditions (1.4) and (1.5) imply integrability of $g$ and $J_{N}$ and for this case a mathematically more satisfactory result was obtained in Sen $\left(1978\right.$, Theorem 4.1). Sen also takes $J_{N}(t)=c_{N, i}$ for $(i-1) / N<t \leqslant i / N$, $i=1, \cdots, N$, and assumes that $J_{N}$ converges pointwise to $J$, but now $J$ is required to be continuous and of bounded variation on closed subintervals of $(0,1)$. This switching of the smoothness condition from $g$ (Wellner) to $J$ (Helmers and Sen) is quite common in problems concerning linear functions of order statistics, where one can use both kinds of smoothness almost interchangeably. The improvement, however, is that instead of (1.4)-(1.6), Sen requires that $g \in L_{q}$ and $\sup _{N}\left\|J_{N}\right\|_{p}<$ $\infty$ for some $p, q \in(1, \infty)$ with $p^{-1}+q^{-1}=1$, to prove that $M_{N} \rightarrow 0$ w.p. 1 . Note that $J_{N} \rightarrow J$ pointwise and $\sup _{N}\left\|J_{N}\right\|_{p}<\infty$ imply $J \in L_{p}$ by Fatou's lemma and together with $g \in L_{q}$ this ensures that Sen's assumption that $J g \in L_{1}$ is automatically satisfied. Apparently unaware of Wellner (1977), Sen also proves another result (Theorem 4.2) which is strictly contained in Wellner's.

The present note constitutes an attempt to provide a mathematically cleaner version of the above results. Roughly speaking we shall show that all smoothness conditions on $g$ and $J$, including (1.6), are superfluous and that the pointwise convergence of $J_{N}$ can be relaxed. We do not assume, that $J_{N}$ is a step function.

2. A strong law. Let $g:(0,1) \rightarrow \mathbb{R}$ be Borel measurable and let $g_{N}$ be defined by (1.1). We begin by proving

Lemma 2.1. With probability $1, g_{N}$ converges to $g$ in Lebesgue measure, i.e., $\lim _{N \rightarrow \infty} \lambda\left\{t:\left|g_{N}(t)-g(t)\right| \geqslant \delta\right\}=0$ for every $\delta>0$. 
Proof. Choose $\varepsilon>0$. By Lusin's theorem there exists a Borel set $B \subset(0,1)$ and a continuous function $\tilde{g}:(0,1) \rightarrow \mathbb{R}$ such that $\lambda(B) \leqslant \varepsilon$ and $g=\tilde{g}$ on $(0,1) \cap$ $B^{c}$. Define $\tilde{g}_{N}(t)=\tilde{g}\left(U_{[N t]+1: N}\right)$ and $B_{N}=\left\{t: U_{[N t]+1: N} \in B\right\}$, so that $g_{N}=\tilde{g}_{N}$ on $(0,1) \cap B_{N}^{c}$. Since $\lambda\left(B_{N}\right)=P_{N}(B)$, where $P_{N}$ denotes the empirical distribution of $U_{1}, \cdots, U_{N}$, it follows from the strong law that $\lim \sup _{N} \lambda\left(B_{N}\right) \leqslant \varepsilon$ w.p. 1. In view of the Glivenko-Cantelli theorem and the continuity of $\tilde{g}$, this implies that w.p. 1 we have for every $\delta>0$

$$
\begin{aligned}
& \lim _{N} \sup \lambda\left\{t:\left|g_{N}(t)-g(t)\right| \geqslant \delta\right\} \leqslant \lambda(B)+\lim _{N} \sup \lambda\left(B_{N}\right) \\
+ & \lim _{N} \sup \lambda\left\{t:\left|\tilde{g}_{N}(t)-\tilde{g}(t)\right| \geqslant \delta\right\} \leqslant 2 \varepsilon .
\end{aligned}
$$

Since $\varepsilon>0$ is arbitrary the lemma is proved.

Theorem 2.1. Let $1 \leqslant p \leqslant \infty, p^{-1}+q^{-1}=1$, and suppose that $J_{N} \in L_{p}$ for $N=1,2, \cdots$ and $g \in L_{q}$. If either

(i) $1<p \leqslant \infty$ and $\sup _{N}\left\|J_{N}\right\|_{p}<\infty$, or

(ii) $p=1$ and $\left\{J_{N}: N=1,2, \cdots\right\}$ is uniformly integrable, then $\lim _{N \rightarrow \infty} M_{N}=0$ with probability 1 .

Proof. Suppose first that $1<p \leqslant \infty$, so $q<\infty$; w.p. $1, g_{N} \rightarrow g$ in Lebesgue measure and $\int\left|g_{N}\right|^{q}=N^{-1} \Sigma\left|g\left(U_{i}\right)\right|^{q} \rightarrow \int|g|^{q}$ by the strong law. By Vitali's theorem this implies that $\int\left|g_{N}-g\right|^{q} \rightarrow 0$, and Hölder's inequality yields $\left|M_{N}\right| \leqslant$ $\left\|J_{N}\right\|_{p}\left\|g_{N}-g\right\|_{q} \rightarrow 0$ w.p. 1 .

Suppose now that $p=1$, so $q=\infty$. Because of the uniform integrability of $J_{N}$ and Lemma 2.1, we have w.p. 1

$$
\begin{aligned}
\lim _{N} \sup \left|M_{N}\right| & \leqslant \delta \lim _{N} \sup \left\|J_{N}\right\|_{1}+2\|g\|_{\infty} \lim _{N} \sup \int_{\left\{\left|g_{n}-g\right|>\delta\right\}}\left|J_{N}\right| \\
& =\delta \lim _{N} \sup \left\|J_{N}\right\|_{1}
\end{aligned}
$$

for every $\delta>0$. Since $\sup _{N}\left\|J_{N}\right\|_{1}<\infty$, the proof is complete.

For $J_{N} \in L_{p}, N=1,2, \cdots$, consider the type of convergence to $J \in L_{p}$ defined by $\lim _{N \rightarrow \infty} \int J_{N} f=\int J f$ for every $f \in L_{q}$. For $1 \leqslant p<\infty$ this is weak convergence in $L_{p}$ and for $p=\infty$ it is weak* convergence in $L_{\infty}$. Necessary and sufficient conditions for a set $\left\{J_{N}, N=1,2, \cdots\right\} \subset L_{p}$ to be sequentially relatively compact in the topology of this convergence are precisely conditions (i) and (ii) in Theorem 2.1 (for $1 \leqslant p<\infty$ see Dunford and Schwartz (1958), IV.8.4 and IV.8.11; for $p=\infty$ see Banach (1932), page 131, for the sufficiency of (i); the necessity is easy). To ensure that $J_{N}$ converges to $J \in L_{p}$ in the above sense one only has to add to conditions (i) and (ii) the further assumption that $\int_{0}^{t} J_{N} \rightarrow \int_{0}^{t} J$ for every $t \in(0,1)$ (see Dunford and Schwartz (1958), IV. 13.23, 25, 27, and Banach (1932), page $135-136)$. Under this additional assumption we may therefore replace $\int J_{N} g$ by $\int J g$ in Theorem 2.1 to obtain

COROLlaRY 2.1. Suppose that the conditions of Theorem 2.1 are satisfied and that there exists a function $J \in L_{p}$ such that $\lim _{N \rightarrow \infty} \int_{0}^{t} J_{N}=\int_{0}^{t} J$ for every $t \in(0,1)$. Then $\lim _{N \rightarrow \infty} \tilde{M}_{N}=0$ with probability 1 . 
Note that the remarks preceding Corollary 2.1 also imply that the conditions on $J_{N}$ and $J$ in the corollary are necessary as well as sufficient to ensure that $\tilde{M}_{N} \rightarrow 0$ w.p. 1 for every $g \in L_{q}$.

3. Variations on a theme. Theorem 2.1 and its corollary clearly contain Wellner's result for $0 \leqslant b_{1}=b_{2}<1$ as well as those of Helmers and Sen (cf. Section 1). In this section we extend our results to cover the other cases discussed by Wellner, which enlarges the range of applications considerably. Though these extensions are straightforward, the conditions inevitably become more cumbersome to state.

For different $b_{1}$ and $b_{2}$ in $[0,1),(1.4)$ and (1.5) allow a different balance between the rates of growth of $g$ and $J_{N}$ near 0 and 1. Correspondingly, we shall show that in Theorem 2.1 and Corollary 2.1 one may allow different values of $p$ and $q$ on different subintervals of $(0,1)$, provided these subintervals overlap; the existence of such overlapping subintervals is easily seen to be equivalent to the assumptions of Theorem 3.1. The indicator function of a set $A$ is denoted by $\chi(A)$ or $\chi(A,$.$) .$

THEOREM 3.1. Let $0=t_{0}<t_{1}<\cdots<t_{k}=1$ and $\varepsilon>0$. For $j=1, \cdots$, $k$, let $1 \leqslant p_{j} \leqslant \infty, p_{j}^{-1}+q_{j}^{-1}=1$ and define intervals $A_{j}=\left(t_{j-1}, t_{j}\right)$ and $B_{j}=\left(t_{j-1}-\varepsilon\right.$, $\left.t_{j}+\varepsilon\right) \cap(0,1)$. Suppose that, for $j=1, \cdots, k, J_{N} \chi\left(A_{j}\right) \in L_{p_{j}}$ for $N=1,2, \cdots$, $g \chi\left(B_{j}\right) \in L_{q_{j}}$ and either

(i) $1<p_{j} \leqslant \infty$ and $\sup _{N}\left\|J_{N} \chi\left(A_{j}\right)\right\|_{p_{j}}<\infty$, or

(ii) $p_{j}=1$ and $\left\{J_{N} \chi\left(A_{j}\right): N=1,2, \cdots\right\}$ is uniformly integrable.

Then $\lim _{N \rightarrow \infty} M_{N}=0$ with probability 1. If, moreover, there exists a function $J$ with $J \chi\left(A_{j}\right) \in L_{p_{j}}$ for $j=1, \cdots, k$, such that $\lim _{N \rightarrow \infty} \int_{0}^{t} J_{N}=\int_{0}^{t} J$ for every $t \in(0,1)$, then also $\lim _{N \rightarrow \infty} \tilde{M}_{N}=0$ with probability 1 .

Proof. Consider an index $j$ with $1<p_{j} \leqslant \infty$, so $q_{j}<\infty$. Choose $\delta \in(0, \varepsilon]$ and define $C_{j}=\left(t_{j-1}-\delta, t_{j}+\delta\right) \cap(0,1)$. The Glivenko-Cantelli theorem and the strong law ensure that w.p. 1

$$
\lim _{N} \sup \int_{A_{j}}\left|g_{N}\right|^{q_{j}} \leqslant \lim _{N} \sup \frac{1}{N} \Sigma\left|g\left(U_{i}\right)\right|^{q_{j}} \chi\left(C_{j}, U_{i}\right)=\int_{C_{j}}|g|^{q_{j}}<\infty .
$$

Since $\delta \in(0, \varepsilon]$ is arbitrary, this implies that $\int\left|g_{N}\right|{ }^{q_{j}} \chi\left(A_{j}\right) \rightarrow \int|g|^{q_{j}} \chi\left(A_{j}\right)$ w.p. 1 by Fatou's lemma. Arguing as in the proof of Theorem 2.1, we conclude that $\int J_{N}\left(g_{N}-g\right) \chi\left(A_{j}\right) \rightarrow 0$ w.p. 1.

For an index $j$ with $p_{j}=1$ and $q_{j}=\infty$, the Glivenko-Cantelli theorem ensures that $\lim \sup _{N}\left\|g_{N} \chi\left(A_{j}\right)\right\|_{\infty} \leqslant\left\|g \chi\left(B_{j}\right)\right\|_{\infty}<\infty$ w.p. 1, and again copying the proof of Theorem 2.1, we find that $\int J_{N}\left(g_{N}-g\right) \chi\left(A_{j}\right) \rightarrow 0$ w.p. 1. This proves the first statement of the theorem. The second statement is obvious because the assumptions of the theorem imply that $\int J_{N} g \chi\left(A_{j}\right) \rightarrow \int \operatorname{Jg} \chi\left(A_{j}\right)$ for $j=$ $1, \cdots, k$.

A second extension of our results concerns, e.g., the case where near a point $t_{0} \in[0,1],|g|$ (or $\left|J_{N}\right|$ ) grows faster than (uniform) integrability would allow, but where the effect of this is cancelled by the fact that $J_{N}$ (or $g$ ) tends to zero at $t_{0}$ at an appropriate rate. Since we are concerned with the product of $J_{N}$ at the point $t$ 
and $g$ at the point $U_{[N t]+1: N}$, this cancellation will work best if we can pin down the order statistics near $t_{0}$ quite close to their expected values. This means that the best results are to be obtained for $t_{0}=0$ and/or 1 , which corresponds to (1.4) and (1.5) for the case where one or both of the $b_{i}$ are outside the interval $[0,1)$. As this is also the most common situation in applications, we shall restrict the few remarks we make to this case. The reader can easily formulate a similar result for arbitrary $t_{0}$ for himself.

Take any $\delta>0$ and define intervals $K_{N, i}$ for $i=1, \cdots,[(N+1) / 2], N=$ $1,2, \cdots$, by

$$
\begin{aligned}
K_{N, i}= & {\left[\frac{i}{N}\left\{\log \left(\frac{N^{2}}{i(N-i+1)}\right)\right\}^{-1-\delta},\right.} \\
& \left.(1+\delta) \frac{i}{N} \log \log \left(\frac{4 N^{2}}{i(N-i+1)}\right)\right] \cap(0,1) .
\end{aligned}
$$

For $P$ - almost every $\omega \in \Omega$, there exists $N(\omega)$ such that for $N \geqslant N(\omega)$ and $i=1, \cdots,[(N+1) / 2]$ we have $U_{i: N} \in K_{N, i}$. This follows easily from Theorems 2 and 3 in Shorack and Wellner (1978) together with Bernstein's inequality for binomial tails. For $N=1,2, \cdots$, define $\bar{g}_{N}:(0,1) \rightarrow[0, \infty]$ by

$$
\begin{aligned}
\bar{g}_{N}(t) & =\sup \left\{|g(s)|: s \in K_{N,[N t]+1}\right\} \quad \text { for } t \in\left(0, \frac{1}{2}\right), \\
& =\sup \left\{|g(1-s)|: s \in K_{N, N-[N t]}\right\} \quad \text { for } t \in\left[\frac{1}{2}, 1\right) .
\end{aligned}
$$

Then, w.p. $1,\left|g_{N}\right| \leqslant \bar{g}_{N}$ on $(0,1)$ for sufficiently large $N$.

For $\eta \in\left(0, \frac{1}{2}\right)$, let $D_{\eta}$ denote the interval $(\eta, 1-\eta)$. The following result is now an immediate consequence of Theorem 3.1.

THEOREM 3.2. Suppose that, for every $\eta \in\left(0, \frac{1}{2}\right), g \chi\left(D_{\eta}\right), J_{N} \chi\left(D_{\eta}\right)$ and $J_{\chi}\left(D_{\eta}\right)$ satisfy the conditions on $g, J_{N}$ and $J$ in Theorem 3.1. If also

$$
\lim _{\eta \rightarrow 0} \lim _{N} \sup \int_{D_{\eta}^{c}}\left|J_{N}\right|\left(\bar{g}_{N}+|g|\right)=\lim _{\eta \rightarrow 0} \int_{D_{\eta}^{c}}|J g|=0
$$

then the conclusions of Theorem 3.1 continue to hold.

\section{REFERENCES}

[1] Banach, S. (1932). Théorie des Opérations Linéaires. Chelsea, New York.

[2] Dunford, N. and Schwartz, J. T. (1958). Linear Operators, Vol. I. Interscience, New York.

[3] Helmers, R. (1977). A strong law of large numbers for linear combinations of order statistics. Report SW 50/77, Mathematisch Centrum, Amsterdam.

[4] SEN, P. K. (1978). An invariance principle for linear combinations of order statistics. Z. Wahrscheinlichkeitstheorie und Verw. Gebiete 42 327-340.

[5] Shorack, G. R. and Wellner, J. A. (1978). Linear bounds on the empirical distribution function. Ann. Probability 6 349-353.

[6] Wellner, J. A. (1977). A Glivenko-Cantelli theorem and strong laws of large numbers for functions of order statistics. Ann. Statist. 5 473-480.

DePaRTMENT OF MATHEMATICS UNIVERSITY OF LEIDEN WASSENAARSEWEG 80 Postbus 95122300 RA LEIDEN The NeTHERLANDS 